\title{
E-Learning y Gamificación: Nuevos modelos de aprendizaje en entornos universitarios ${ }^{1}$
} Amparo Maset-Llaudes ${ }^{a}$, David Cabedo ${ }^{b}$

${ }^{a}$ Departmento de Finanzas y Contabilidad, Universitat Jaume I, Castellón, España, maset@uji.es bepartmento de Finanzas y Contabilidad, Universitat Jaume I, Castellón, España, cabedo@uji.es

\section{Resumen}

La incorporación de los dispositivos electrónicos a los tradicionales métodos de aprendizaje ha dado lugar a la llamada e-learning, que en el presente trabajo se considera como una iniciativa enfocada a mejorar el proceso de aprendizaje en educación superior a través del uso de dispositivos electrónicos en las aulas. Esta iniciativa se basa en un concepto cada vez más destacado en el campo de la enseñanza: la gamificación. El uso de software especifico de aprendizaje en las aulas a través de los dispositivos electrónicos personales se ha expandido últimamente al amparo del desarrollo de las nuevas tecnologías, las cuáles se han incorporado a la actividad cotidiana presencial en las asignaturas universitarias. Se considera que estas nuevas técnicas facilitan el aprendizaje al derivar en una mayor implicación activa de los alumnos en las clases presenciales.

Este trabajo pretende analizar cuáles son los efectos de la incorporación de nuevo software específico de aprendizaje en las clases de la asignatura Análisis de los Estados Financieros, de carácter obligatorio y de segundo curso del grado de Administración de Empresas, Economía y Finanzas y Contabilidad. Para ello se utilizarán los resultados de la evaluación de dicha asignatura con el fin de determinar en qué medida la utilización del software "Socrative" puede o no afectar a estos resultados, es decir, para dilucidar si la participación en este programa de aprendizaje mejora las habilidades y resultados de los estudiantes. Se espera que su participación en este programa de mejora educativa tenga un impacto neto positivo en la calificación de la asignatura. El trabajo presenta los resultados del grupo-

\footnotetext{
${ }^{1}$ Este trabajo ha sido realizado con el apoyo de la ayuda procedente de la "Convocatòria d'ajudes a la innovació educativa de la Universitat Jaume I del curso 2017/18” (Proyecto de Innovación Educativa, Referencia 3498).
} 
piloto con el que se inició el estudio, así como una comparativa con los resultados del resto de grupos y la descripción de los siguientes pasos que están en proceso de implementación en esta propuesta de mejora educativa.

Palabras clave: e-Learning, gamificación, Socrative, estudios universitarios.

\section{Introducción}

El e-learning es una modalidad de aprendizaje que se basa en el uso de nuevas tecnologías. Este trabajo presenta un ejemplo de e-learning considerado como una iniciativa enfocada a mejorar el proceso de aprendizaje en educación superior. Esta iniciativa se desarrolla tomando como base un concepto convertido en fundamental en los últimos años en el campo de la enseñanza: la gamificación (Glover, 2013; Topal y Karaca, 2018). El uso de software en las aulas que facilita la asunción de los contenidos de cualquier asignatura se ha expandido al amparo del desarrollo de las nuevas tecnologías, las cuáles se han incorporado a la actividad cotidiana presencial en las asignaturas universitarias. Se considera que estas nuevas técnicas facilitan el aprendizaje al derivar en una mayor implicación activa de los alumnos en las clases presenciales (Cheung y Vogel, 2013; Gros y Garcia-Peñalvo; 2016 Sife et al, 2007). Este trabajo pretende analizar cuáles son los efectos del uso continuo de estas prácticas en las clases de la asignatura Análisis de los Estados Financieros, asignatura obligatoria de segundo curso del grado de Administración de Empresas, Economía y Finanzas y Contabilidad. Para ello se utilizarán los resultados de la primera convocatoria de la asignatura AEF del curso 2017/2018, con el fin de determinar en qué medida la utilización del software Socrative puede o no afectar a estos resultados, es decir, para dilucidar si la participación en este programa de mejora educativa mejora las habilidades y resultados de los estudiantes.

Dado que en la actualidad la práctica totalidad del estudiantado cuenta con dispositivos móviles de uso personal, en esta asignatura se ha optado por utilizar este medio para realizar actividades prácticas en el aula. Dichas actividades se pueden llevar a cabo a través de teléfonos móviles, tablets o incluso ordenadores portátiles. Es lo que se conoce como Bring Your Own Devide (BYOD) o Bring Your Own Technology (BYOT) (Lim, 2017). Este tipo de actividades se enmarca en lo que se conoce como mobile- learning o mlearning (Shippee y Keengwe, 2012) Durante este curso, los alumnos que han procedido a realizar la actividad no han mostrado predilección por ninguno de estos instrumentos, habiéndose observado la presencia indistinta de todos ellos en el aula durante la realización de dicha actividad. 


\section{La gamificación en el aula: el programa Socrative en la asignatura Análisis de Estados Financieros}

El uso de dispositivos móviles en el aula cada vez ha ido ganando más adeptos, de manera que se ha ido generalizando a medida que se ha ido incorporando a la actividad ordinaria de la mayoría de los individuos. El mundo educativo no ha sido ajeno a esta evolución, y paulatinamente ha ido incorporando a su actividad habitual tanto software específico como hardware, siendo generalizado el uso de ordenadores y tablets en todos los niveles de enseñanza (Dakka, 2015).

En el trabajo que aquí se presenta, se va a analizar el uso de una conocida herramienta de gamificación durante el desarrollo de las clases que permite al alumnado interactuar con el profesor, al proporcionar éste a través de dicho programa diversas actividades a realizar en el aula (Coca y Slisko, 2013; Kaya y Balta, 2016). Se trata de Socrative, uno de los programas más utilizados de software gratuito (Tretinjak et al, 2015; Wash, 2014), usado de manera presencial, es decir, aunque se podría llevar a cabo la actividad a distancia, esta opción no ha sido considerada dado que para acceder a dicha actividad se necesita una clave que el profesor proporciona exclusivamente a los alumnos con presencia en el aula (number room). La actividad, que consiste en la resolución de un test (Quiz) de 20 preguntas con respuesta múltiple (cuatro respuestas de las cuales una es la correcta), se llevó a cabo durante las clases prácticas, aunque sus cuestiones abarcan tanto preguntas prácticas como teóricas. A pesar de tratarse de preguntas tipo test y por lo tanto muy escuetas, el programa permite que los alumnos tengan acceso a una explicación sobre por qué las repuestas son correctas o erróneas, una vez hayan contestado la pregunta, con lo cual el feedback obtenido por el alumno es inmediato.

Una vez respondido el test, el profesor obtiene información no sólo sobre la nota o nivel de respuestas acertadas alcanzado por el alumno, sino también sobre qué cantidad de alumnos han respondido correctamente a cada pregunta, teniendo de esta forma una clasificación del nivel de dificultad de cada una de las cuestiones.

Aunque existen diferentes opciones con respecto a las características del test, en esta ocasión el proceso de respuesta no ha estado limitado por el tiempo (los alumnos han tenido todo el tiempo necesario para contestar las preguntas) y, en aras de poder establecer una comparación lo más objetiva posible, todos ellos han tenido formuladas, tanto las preguntas como las respuestas, en el mismo orden. Las preguntas son respondidas por parte del estudiante de una forma automática, sin intervención alguna del profesor (más allá, claro está, de la programación de la actividad).

El programa Socrative ofrece una variedad de preguntas a elegir (respuesta múltiple, verdadero/falso y preguntas cortas) sin embargo, la elección de preguntas tipo test de respuesta múltiple obedece a que los exámenes finales de esta asignatura se dividen en tres 
partes: un ejercicio contable largo, una serie de preguntas cortas con cálculos sobre fórmulas y comentarios sobre los resultados, y preguntas tipo test. Estas últimas son iguales a las utilizadas en las actividades presenciales del Socrative, y las preguntas de la segunda parte también se pueden incorporar a la actividad, de manera que el $60 \%$ de la nota de examen se ve cubierta por la actividad realizada con este programa durante el curso.

Aunque se puede optar por un entorno de trabajo colaborativo al poder formar los estudiantes grupos de trabajo, en esta ocasión se ha decidido que los estudiantes, de manera individual, respondan a los test por su semejanza con una parte importante de los exámenes finales.

\section{La asignatura Análisis de Estados Financieros}

En este trabajo se pretende analizar la efectividad de la incorporación de un programa informático de aprendizaje a las actividades habituales de las clases presenciales de la asignatura Análisis de Estados Financieros. Para ello se ha comparado el conjunto de alumnos que estudiaron esta asignatura mediante el uso del programa Socrative, con aquellos que no lo utilizaron en ningún momento a lo largo del curso. Dado el nivel de detalle de la asignatura, esto no supuso ningún problema adicional, al recibir todos la misma materia impartida de la misma forma.

La asignatura Análisis de Estados Financieros se imparte en la Universitat Jaume I de Castellón en el segundo curso, segundo semestre, de los grados de Economía, Administración y Dirección de Empresas y Finanzas y Contabilidad de la Universidad Jaume I. La asignatura tiene 7 grupos de teoría y 12 grupos de práctica, impartiéndose la asignatura en inglés en un solo grupo de cada clase.

\section{Objetivos y desarrollo de la actividad}

El objetivo del presente trabajo es valorar la implementación de un sistema de actividad en el aula que utilice instrumentos basados en la formación más que en la calificación, que implique a los estudiantes en un proceso de aprendizaje reflexivo y continuo y que implemente técnicas que proporcionen un feedback eficaz y oportuno en el tiempo que les permita "practicar" y anticipar parte de las preguntas a las que se enfrentan en el examen final de la asignatura. Para ello se valoran los resultados de una prueba piloto llevada a cabo en el curso 2017/18 en la asignatura Análisis de los Estados Financieros.

Una parte de la calificación final de la asignatura (40\%) se obtiene mediante la resolución de preguntas tipo test (entre 6 y 10 preguntas) y preguntas cortas de cálculo y comentario de resultados (entre tres y siete). El resto de la asignatura se divide entre en ejercicio 
práctico $(30 \%)$ y un trabajo a realizar por el alumno bien individualmente $(30 \%)$. Se ha realizado un prueba piloto dirigida a proporcionar a los alumnos experiencia previa sobre la resolución de pregunta tipo test, utilizando durante las clases prácticas de la asignatura el software Socrative, que permite que los alumnos respondan a preguntas similares a las que se van a encontrar posteriormente en el examen y que reciban un feedback inmediato de cada respuesta sin la intervención del profesor. Ese feedback inmediato no solamente va a consistir en la información sobre si la respuesta dada por el alumno es correcta o incorrecta, si no que incorpora la explicación de porqué cada respuesta múltiple es errónea o no lo es. El profesor previamente podrá redactar el feedback que considere necesario para que los alumnos entiendan las explicaciones proporcionadas sobre las respuestas.

Dado que se pretende es que los alumnos practiquen esta actividad, y que vayan asimilando la comprensión de cada pregunta, se les advierte que las respuestas erróneas no restarán puntuación, al contrario de lo que ocurre en el examen final, en el que las respuestas mal contestadas restan un $25 \%$ de la nota obtenida en el test.

\section{Parte empírica. Planteamiento}

Objetivos

1. Implicar a los estudiantes en un proceso de aprendizaje reflexivo y continuo

2. Utilizar instrumentos de evaluación basados en la formación más que en la calificación.

3. Implementar técnicas de seguimiento individualizado que proporcionen un feedback eficaz y oportuno en el tiempo

Características de la asignatura:

1. Análisis de los Estados Financieros, asignatura de segundo curso, segundo semestre, común para los grados de Economía, Administración y Dirección de Empresas y Finanzas y Contabilidad

2. Curso: $2017 / 2018$

3. Estudiantes matriculados: 287

4. Profesores: 6

5. Grupos de teoría: 7; Grupos de práctica: 12

6. Calificación: $70 \%$ examen final escrito (30\% caso práctico largo, 20\% preguntas cortas a comentar, $20 \%$ preguntas test), 30\% elaboración de un trabajo final práctico 
Prueba piloto test respuesta múltiple:

1. ¿Qué se evalúa?: test de respuesta múltiple en Socrative como actividad continua

2. Profesores que participan: 2

3. Grupos que participan: grupos de práctica: 2

4. Tamaño de la muestra: 57

Variables

1. Supera o no la asignatura (Supera)

2. Calificación de la asignatura (Nota final)

3. Créditos superados por el alumno (Créditos superados)

4. Media del expediente (Media expediente)

5. Créditos troncales superados por el alumno Créditos troncales)

6. Pertenencia del alumno al grado de Administración de Empresas (ADE)

7. Pertenencia del alumno al grado de Economía (ECO)

8. Pertenencia del alumno al grado de Finanzas y Contabilidad (FICO)

9. Pertenencia del alumno al grupo Erasmus (Erasmus)

10. Uso de Socrative en clase (Socrative)

Software utilizado

Para la realización de la prueba piloto se ha utilizado el programa gratuito Socrative, con la modalidad de preguntas tipo test. El software permite conocer a los alumnos en el momento en que responden si la contestación proporcionada ha sido o no correcta, y en este caso, obtienen información sobre cuál sería la respuesta correcta y por qué, sin intervención alguna del profesor en ese momento.

\section{Parte empírica. Datos y resultados}

En el trabajo se analiza la participación de los alumnos en la actividad llevada a cabo durante la clase de prácticas consistente en la realización de test de respuesta múltiple con una sola respuesta correcta, comparando sus resultados obtenidos en los exámenes finales con los de aquellos que no realizaron la actividad.

En un primer análisis empírico realizado mediante el test ANOVA, se ha testado si existen diferencias en las notas finales obtenidas por los alumnos. En concreto, la hipótesis básica a testar es si existen diferencias significativas entre los valores medios de las notas finales de 
la asignatura entre los alumnos que realizaron la actividad mediante el Socrative y aquellos que no realizaron dicha actividad. Los resultados pueden comprobarse en la siguiente tabla.

Tabla 1. Anova

\begin{tabular}{|c|c|c|c|c|c|}
\hline & $\begin{array}{l}\text { Suma de } \\
\text { cuadrados }\end{array}$ & gl & $\begin{array}{c}\text { Media } \\
\text { cuadrática }\end{array}$ & $\mathbf{F}$ & Sig. \\
\hline Entre grupos & 36,444 & 1 & 36,444 & 7,219 & ,016 \\
\hline $\begin{array}{l}\text { Dentro de } \\
\text { grupos }\end{array}$ & 85,826 & 17 & 5,049 & & \\
\hline Total & 122,269 & 18 & & & \\
\hline
\end{tabular}

Fuente: elaboración propia a partir de los datos de la muestra

Como puede observarse, dado el nivel de significatividad obtenido, se considera que sí que existen diferencias significativas entre los valores medios de las calificaciónes obtenidas por los alumnos entre los que realizaron la actividad y los que no lo hicieron, es decir, se acepta la hipótesis nula indicada anteriormente.

En un segundo nivel de análisis se ha procedido a estimar dos modelos econométricos. En primer lugar (Modelo 1), un modelo logit en el que la variable independiente binaria será haber superado o no la asignatura. En segundo lugar, se ha estimado un modelo de regresión lineal (Modelo 2) en el que la variable dependiente es la nota obtenida por el alumno en el examen final. En ambos modelos se ha incorporado la variable que refleja el uso de Socrative en las aulas como variable independiente, con el fin de poder analizar cuál es su influencia tanto sobre la calificación del alumno como sobre la probabilidad de aprobar la asignatura. 
Tabla 2. Modelos logit y regresión lineal

\begin{tabular}{|l|c|c|}
\hline Convocatoria & $-1,631$ &,- 749 \\
\hline Erasmus & $(0,106)$ & $(0,509)$ \\
\hline ADE & $1,111 \mathrm{E}+02$ & 3,043 \\
\hline ECO & $(0,999)$ & $-0,467$ \\
\hline Créditos Superados & 1,821 & 0,637 \\
\hline Créditos troncales & $(0,135)$ & $-0,778$ \\
\hline Media expediente & 2,951 &, 813 \\
\hline Socrative & $(0,035)^{*}$ & $(0,724)$ \\
\hline R2 Ajustado &, 154 & 0,00048 \\
\hline (Constante) & $(0,996)$ & $(0,114)$ \\
\hline Logaritmo de la verosimilitud -2 &,- 153 & \\
\hline R cuadrado de Cox y Snell & $(0,997)$ &, 013 \\
\hline R cuadrado de Nagelkerke & $(0,157)$ & $(0,054)^{* *}$ \\
\hline Significativo al 10\% & 3,314 &,- 524 \\
\hline & $(0,067)^{* *}$ & $(0,807)$ \\
\hline & $-127,982$ & $-7,682$ \\
\hline & 30,988 & 0,654 \\
\hline & 0,571 & 0,434 \\
\hline
\end{tabular}

Fuente: elaboración propia a partir de los datos de la muestra

Según los resultados del primer modelo, el uso de la herramienta Socrative afecta positivamente a la probabilidad de aprobar la asignatura, resultando esta variable significativa, así como la pertenencia del alumno al grado de Economía, siendo también positiva la influencia de las variables pertenencia al grupo Erasmus y al grado de Administración de Empresas, créditos superados y la media del expediente, aunque estas variables no resultan ser significativas.

Con respecto al segundo modelo en el que la variable dependiente es la nota obtenida en el examen final, el signo de la variable correspondiente al uso del programa Socrative no resulta ser el esperado, al aparecer ésta con signo negativo en la ecuación, aunque dicha variable ha resultado no ser significativa. En esta ocasión la única variable que es 
significativa ha sido la media del expediente, que cuenta con el signo positivo esperado. El resto de variables afectan todas positivamente a la nota final del alumno aunque no resultan ser significativas. La única variable que aparece con signo negativo en ambos modelos es la variable Convocatoria, indicando que a más convocatorias es menor la probabilidad de aprobar, siendo así su relación con la nota final de la asignatura inversa. Ello puede deberse a la dificulatad que entraña para un alumno que ha pasado a cursos superiores estudiar la asignatura al no poder acudir a las clases presenciales habitualmente debido problemas de solapamientos de horarios, destacando de esta manera la importancia de la presencia en las clases y del seguimiento continuo de las mismas por parte de los alumnos de Análisis de Estados Financieros.

\section{Conclusiones}

La gamificación en entornos universitarios se está extendiendo paulatinamente, por lo que es de todo punto necesario valorar cuál es su incidencia sobre los resultados de los alumnos, con el fin de determinar si su inclusión como herramienta habitual de trabajo en las aulas es beneficioso para el desarrollode la asignatura y provoca la mejora del aprendizaje del alumnado.

Según los resultados obtenidos, podemos afirmar en primer lugar que sí que existen diferencias entre aquelos alumnos que utilizaron el programa Socrative en la asignatura Análisis de Estados Financieros a lo largo de cada uno de los temas. Con respecto a los resultados obtenidos se puede afirmar que la implicación en el proyecto piloto por parte de los alumnos con la utilización de la herramienta Socrative, aunque no parece que tenga la incidencia esperada sobre el nivel de calificación obtenido, sí que mejora la probabilidad de aprobar la asignatura.

Cabría destacar que esta es un aexperiencia piloto que se ha llevado a cabo por primera vez en el curso 2017/2018. Una vez extendida durante los próximos cursos académicos al resto de los alumnos, se espera que su influencia sea positiva tanto sobre la probabilidad de aprobar como sobre la nota final obtenida en la asignatura que nos ocupa. 


\section{Referencias}

Cheung, R., \& Vogel, D. (2013). Predicting user acceptance of collaborative technologies: An extension of the technology acceptance model for e-learning. Computers \& Education, 63, 160175 .

Coca, D. M., \& Sliško, J. (2017). Software Socrative and smartphones as tools for implementation of basic processes of active physics learning in classroom: An initial feasibility study with prospective teachers. European Journal of Physics Education, 4(2), 17-24.

Dakka, S. M. (2015). Using Socrative to enhance in-class student engagement and collaboration. International Journal on Integrating Technology in Education (IJITE), 4(3), 13-19.

Glover, I. (2013). Play as you learn: gamification as a technique for motivating learners. In EdMedia: World Conference on Educational Media and Technology (pp. 1999-2008). Association for the Advancement of Computing in Education (AACE).

Gros, B., \& García-Peñalvo, F. J. (2016). Future trends in the design strategies and technological affordances of e-learning. Learning, Design, and Technology: An International Compendium of Theory, Research, Practice, and Policy, 1-23.

Kaya, A., \& Balta, N. (2016). Taking advantages of technologies: using the Socrative in English language teaching classes. International Journal of Social Sciences \& Educational Studies, 2(3), 4-12.

Lim, W. N. (2017). Improving student engagement in higher education through mobile-based interactive teaching model using socrative. IEEE Xplore Digital Library, 404-412.

Doi:10.1109/EDUCON.2017.7942879.

Shippee, M., \& Keengwe, J. (2014). mLearning: Anytime, anywhere learning transcending the boundaries of the educational box. Education and Information Technologies, 19(1), 103-113.

Sife, A., Lwoga, E., \& Sanga, C. (2007). New technologies for teaching and learning: Challenges for higher learning institutions in developing countries. International journal of education and development using ICT, 3(2), 57-67.

Topal, M., \& Karaca, O. (2018). Gamification in E-Learning. In Emerging Trends, Techniques, and Tools for Massive Open Online Course (MOOC) Management (pp. 79-105). IGI Global.

Tretinjak, M. F., Bednjanec, A. \& Tretinjak, M. (2015). Interactive teaching with Socrative. 38th International Convention on Information and Communication Technology, Electronics and Microelectronics, MIPRO 2015 - Proceedings, 848-851.

Wash, P. (2014). Taking advantage of mobile devices: Using Socrative in the classroom. Journal of Teaching and Learning With Technology, 3(1), 99-101. https://doi.org/https://doi.org/10.14434/jotlt.v3n1.5016 